\title{
Determination of Trace Rare Earth Impurities in Tantalum Pentaoxide by Electrothermal Vaporization ICP-MS Using in situ Volatilization for Matrix Removal
}

\author{
${ }^{a}$ Shizhong Chen, ${ }^{b}$ Shengping Zhu, and a Dengbo Lu \\ ${ }^{\mathrm{a} C o l l e g e}$ of Chemical and Environmental Engineering, Wuhan Polytechnic University, \\ Wuhan 430023, P. R. China \\ 'bepartment of Chemistry, Yunyang Teacher' College, Danjiangkou 442700, Hubei, P.R.China
}

\section{INTRODUCTION}

As a basic substance for advanced functional and structural materials, tantalum pentaoxide is widely used in the electrical, mechanical, steel, optical, aviation, and semiconductor industries owing to its excellent properties (1, 2 ). The properties and applications of high purity materials in the hightech fields depend not only on the characteristics of the basic substances, but also on the purity of the compounds $(3,4)$. Thus, the development of a rapid, sensitive, accurate, and direct method for the determination of trace impurities in high purity $\mathrm{Ta}_{2} \mathrm{O}_{5}$ is an important research topic.

The most common methods for the determination of trace impurities in high purity materials are inductively coupled plasma atomic emission spectrometry (ICP-AES), inductively coupled plasma mass spectrometry (ICP-MS), and atomic absorption spectrometry (AAS) (510). However, the conventional methods for the determination of trace impurities in high purity materials may suffer from insufficient sensitivity and matrix effects, especially spectral interferences from the line-rich spectrum of Ta. Therefore, separation and pre-concentration techniques, such as liquid-liquid extraction, coprecipitation, ion exchange, electrodeposition and chromatography, are generally required prior to the

*Corresponding author.

E-mail: chenshizbong62@163.com

Fax: +862783956442

\begin{abstract}
In this paper, an in situ matrix separation method has been developed for the determination of trace rare earth impurities in high purity tantalum pentaoxide $\left(\mathrm{Ta}_{2} \mathrm{O}_{5}\right)$ by electrothermal vaporization inductively coupled plasma mass spectrometry (ETV-ICP-MS). A polytetrafluoroethylene (PTFE) slurry was used as a chemical modifier to convert both the matrix (Ta) and the rare earth elements (REEs) into their fluorides, with different volatilities, at high temperature in a graphite furnace. The more volatile $\mathrm{TaF}_{5}$ was removed in situ by selective vaporization prior to the determination of the analytes, eliminating matrix effects.

Under optimum operating conditions, the detection limits (DLs) for $\mathrm{La}, \mathrm{Eu}$, and $\mathrm{Yb}$ were found to be 4.2, 1.9, and $3.5 \mathrm{ng} \mathrm{g}^{-1}$, with relative standard deviations (RSDs) less than 5.0\%. The recommended method was successfully applied to the determination of trace impurities (La, $\mathrm{Eu}$, and $\mathrm{Yb}$ ) in $\mathrm{Ta}_{2} \mathrm{O}_{5}$, and the results were in good agreement with those obtained by pneumatic nebulization (PN) ICP-MS after separation of the matrix using a solvent extraction procedure.
\end{abstract}

determination for the elimination of matrix interferences and improvement of analytical sensitivity (1115). However, these additional steps could lead to low sample throughput, risk of contamination, loss of analyte, and discharge of pollutants from sample pretreatment. Electrothermal vaporization (ETV) as a sampling technique could offer the following advantages: (a) small sample requirement, (b) high transport efficiency, (c) low absolute detection limits, and (d) direct analysis of solid samples. Furthermore, the application of various chemical modifiers in ETV not only enhances the analytical sensitivity for refractory and carbide-forming elements (such as $\mathrm{Zr}, \mathrm{V}$, Ti, Cr, $\mathrm{Al}$, and rare earth elements), but also effectively separates the matrix constituents from the analytes by suitable selection of furnace temperature programs. The representative examples are the application of halogenating reagents, metal salts, alkylating reagents, and chelating reagents (16-22). Among the chemical modifiers, a polytetrafluoroethylene (PTFE) slurry has drawn growing attention owing to the following attractive features: (a) high fluorine content, (b) sufficient activity to react with refractory material, (c) suitably high decomposition temperature (about $415^{\circ} \mathrm{C}$ ), (d) few inorganic impurities, and (e) convenient use. PTFE has been applied for the direct analysis of high purity and refractory materials $(23,24)$.

The present work is aimed at converting both the matrix (Ta) and the rare earth elements (REEs) in $\mathrm{Ta}_{2} \mathrm{O}_{5}$ into their corresponding fluorides, characterized with different volatilities, at a high temperature in a graphite furnace using a polytetrafluoroethylene (PTFE) slurry as a fluorinating reagent. Prior to the transport of the analytes to the plasma, the more easily volatile tantalum fluoride 
(boiling point: $230^{\circ} \mathrm{C}$ ) can be vaporized and separated in situ from the fluorides of the analytes (boiling points: about $2200^{\circ} \mathrm{C}$ ) in the ashing step. Based on the facts mentioned above, a novel method for the analysis of $\mathrm{Ta}_{2} \mathrm{O}_{5}$ via in situ separation of the matrix from the analytes has been developed by ETV-ICP-MS. The proposed method is characterized by simplicity, speed, small sample requirement, and limited chemical pretreatment.

\section{EXPERIMENTAL}

\section{Instrumentation}

An X-7 ICP-MS system (Thermo Elemental Corporation, USA), equipped with a modified commercially available WF-4C graphite furnace (Beijing Analysis Instrument Factory, Beijing, P.R. China) as an electrothermal vaporizer, was used for the determination of the analytes. The optimum operating conditions of ETV-ICP-MS are summarized in Table I. A Model Ethos T microwave system (Milestone, Italy) was used for sample digestion.

\section{Reagents}

The standard solutions (1.0 $\mathrm{mg} \mathrm{mL}^{-1}$ ) of $\mathrm{La}, \mathrm{Eu}$, and $\mathrm{Yb}$ were prepared by dissolving their high purity oxides in $2.0 \mathrm{~mol} \mathrm{~L}^{-1}$ $\mathrm{HNO}_{3}$, and made up to $25.0 \mathrm{~mL}$ volume with doubly distilled water. A $60 \%(\mathrm{~m} / \mathrm{v})$ PTFE emulsion $(\mathrm{d}<1 \mu \mathrm{m}$, viscosity $7 \times 10^{-3}-15 \times 10^{-3} \mathrm{~Pa}$ s) was purchased from the Shanghai Organic Chemistry Institute (Shanghai, P.R. China). All other reagents were of analytical grade or better. High purity deionized water (18.2 $\mathrm{M} \Omega$ ) obtained from Milli-Q ${ }^{\mathrm{TM}} \mathrm{A} 10$ system (Millipore Corporation, USA) was used throughout this work.

\section{Sample Preparation}

A sample portion of $0.1000 \mathrm{~g}$ $\mathrm{Ta}_{2} \mathrm{O}_{5}$ (Shanghai Reagent Factory, Tianjin, P.R. China) was accurately weighed into a 100-mL Teflon ${ }^{\circledR}$ microwave digestion vessel, to which $2.0 \mathrm{~mL}$ of concentrated $\mathrm{HNO}_{3}$ and $3.0 \mathrm{~mL}$ concentrated HF were added. The samples were heated in a microwave oven at $200{ }^{\circ} \mathrm{C}$ (ramp: 10 minutes, hold: 30 minutes) at $1.0 \mathrm{~kW}$ power. After the digestion had been completed, the vessels were cooled to room temperature and vented carefully. The resulting solutions were transferred to a PTFE beaker and heated to near dryness for removal of HF. The residues were dissolved in $2.0 \mathrm{~mL}$ of $0.1 \mathrm{~mol} \mathrm{~L}^{-1} \mathrm{HNO}_{3}$. Next, $0.10 \mathrm{~mL}$ of $60 \%$ (w/v) PTFE slurry was added, followed by dilution to $4.0 \mathrm{~mL}$ with deionized water. The resultant mixture was dispersed with an ultrasonic vibrator for 15 minutes before use. Aqueous standard solutions containing $6 \%(\mathrm{w} / \mathrm{v})$ PTFE were used for calibration. The blank was prepared in exactly the same way as the sample.

\section{Recommended Procedure}

After the ETV unit was connected to the ICP-MS and the system stabilized, a $10 \mu \mathrm{L}$ volume of sample was injected into the graphite furnace. The sample inlet hole was blocked with a graphite cone. After being dried and ashed, the analyte was vaporized and carried into the plasma by the carrier gas (argon) for ICP-MS determination.

\section{RESULTS AND DISCUSSION}

\section{Investigation of Vaporization Behavior of Analytes}

Rare earth elements (REEs) are difficult to vaporize and determine directly by conventional ETV-ICPMS, even at a high temperature, because the boiling points of both the REEs and their oxides are above $3000{ }^{\circ} \mathrm{C}$, which is much higher than the $2800{ }^{\circ} \mathrm{C}$ commonly provided by conventional ETV. In order to solve this problem, application of a chemical modifier to form a more volatile species is an
TABLE I

Operating Parameters for ETV-ICP-MS

\begin{tabular}{ll}
\hline ICP-MS Plasma & X-7 ICP-MS system \\
Plasma power & $1300 \mathrm{~W}$ \\
Plasma argon flow rate & $14.5 \mathrm{~L} \mathrm{~min}^{-1}$ \\
Auxiliary argon flow rate & $0.78 \mathrm{~L} \mathrm{~min}^{-1}$ \\
Nebulizer argon flow rate & $0.95 \mathrm{~L} \mathrm{~min}^{-1}$ \\
Sampler orifice (nickel) & $1.1 \mathrm{~mm}$ \\
Skimmer orifice (nickel) & $0.7 \mathrm{~mm}$ \\
Acquisition mode & Peak-jumping \\
Number of sweeps & 100 \\
Dwell time & $10 \mathrm{~ms}$ \\
Acquisition time & $40 \mathrm{~s}$ \\
Number of measurements per peak & 3 \\
Isotopes & $139 \mathrm{La}, 153 \mathrm{Eu}, 172 \mathrm{Yb}$, and $181 \mathrm{Ta}$ \\
ETV ---- & $10 \mu \mathrm{L}$ \\
Sample volume & $0.50 \mathrm{~L} \mathrm{~min}{ }^{-1}$ \\
Carrier gas flow rate & $100^{\circ} \mathrm{C}$, ramp: $10 \mathrm{~s}$, hold: $20 \mathrm{~s}$ \\
Drying temperature & $600^{\circ} \mathrm{C}$, ramp: $10 \mathrm{~s}$, hold: $50 \mathrm{~s}$ \\
Ashing step & $2600^{\circ} \mathrm{C}$, hold: $4 \mathrm{~s}$ \\
Vaporization step &
\end{tabular}


effective way to increase the sample introduction efficiency and to improve the analytical performance. Figure 1 shows the typical signal profiles of $\mathrm{Yb}$ with and without PTFE. In the presence of PTFE, a sharper and more intense signal from $\mathrm{Yb}$ was detected without memory effect $(a, b)$, while the contrary is observed in the absence of PTFE $(c, d)$. Similar results were also obtained for $\mathrm{Ta}$ and the other REEs. The results mentioned above can be explained by the fact that the ETV temperature is not high enough to vaporize the refractory elements. However, when using PTFE as a chemical modifier, its decomposition products can strongly react with the refractory elements to form the corresponding fluorides whose boiling points are lower than those of their metals, oxides, or carbides, thus leading to an improved vaporization and transport efficiency. In addition, Figure 1 also gives the signal of the blank solution (e).

\section{In situ Separation of Matrix from Analytes}

Due to the different volatility of tantalum fluoride (matrix) with REE fluorides (analytes), a rational selection of ashing temperature and time would be the key to in situ separation of the matrix from the analytes in the graphite furnace. Therefore, the effect of ashing temperature and time on the signal intensities of the matrix and analytes was investigated with PTFE (shown in Figures 2 and 3), in which $\mathrm{La}, \mathrm{Eu}$, and $\mathrm{Yb}$ were chosen as the representatives of the light, medium, and heavy REEs, respectively. Figure 2 shows that in the presence of PTFE, the signal loss of the matrix (Ta) occurs at $500^{\circ} \mathrm{C}$, and Ta can be completely evaporated with an increase in ashing temperature. This indicates that the active fluorine radicals produced by the decomposition of the PTFE reacted with Ta to form $\mathrm{TaF}_{5}$, which is highly volatile (b.pt. $230^{\circ} \mathrm{C}$ ) and can be removed quickly from the graphite furnace by the carrier gas. On the other hand, the ashing losses of the REEs do not appear until the ashing temperature rises to above $1600^{\circ} \mathrm{C}$. These facts indicate that the Ta matrix can be separated in situ from the REEs in the graphite furnace by selecting a suitable ashing temperature.

\section{Atomic Apectroscopy Vol. 34(1), Jan../Feb. 2013}

In addition, the results shown in Figure 3 show that the analytes' signal intensities do not decrease by prolonging the ashing time at the selected ashing temperature. On the contrary, the signal intensity of the matrix (Ta) decreases significantly owing to the formation of the highly volatile $\mathrm{TaF}_{5}$. Based on the above experimental result, it appears possible to remove the matrix (Ta) by selecting an appropriate ashing temperature and time prior to vaporization of the analytes. In this work, an ashing temperature of $600{ }^{\circ} \mathrm{C}$ and an ashing time of 50 seconds were used for the following experiments. Under these conditions, the Ta matrix could be mostly removed, while the analyte still remained in the graphite tube without considerable loss.

\section{Effect of Vaporization Temperature}

The dependence of the signal intensities of the analytes on the vaporization temperature was investigated. It can be seen from Figure 4 that in the presence of PTFE, stronger signal intensities were detected, and reached a

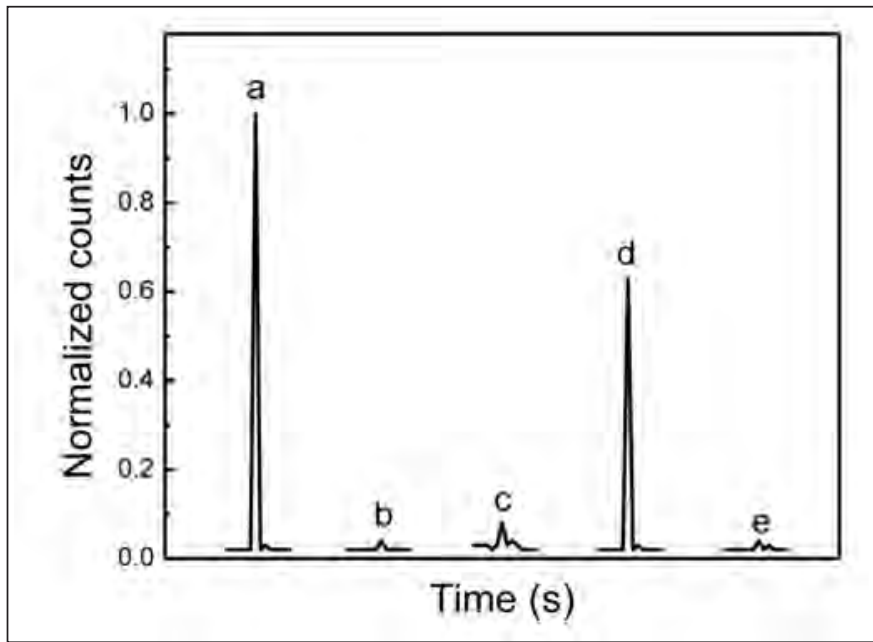

Fig. 1. Typical signal profiles for $\mathrm{Yb}$. (a) $3.0 \mathrm{ng} \mathrm{mL}^{-1}$ of $\mathrm{Yb}$ with PTFE, (b) residual signal from a with PTFE, (c) $10 \mathrm{ng} m L^{-1}$ of $Y b$ without PTFE, (d) residual signal from (c) with PTFE, and (e) signal from blank.

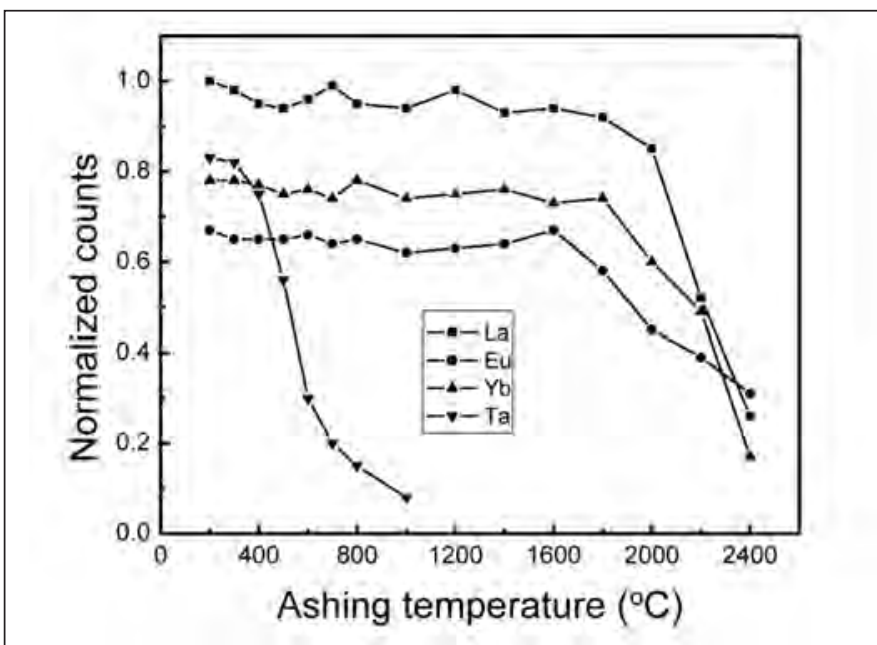

Fig. 2. Dependence of signal intensity on ashing temperature. With PTFE: La, Eu, Yb. and Ta, $3.0 \mathrm{ng} m L^{-1}$. 


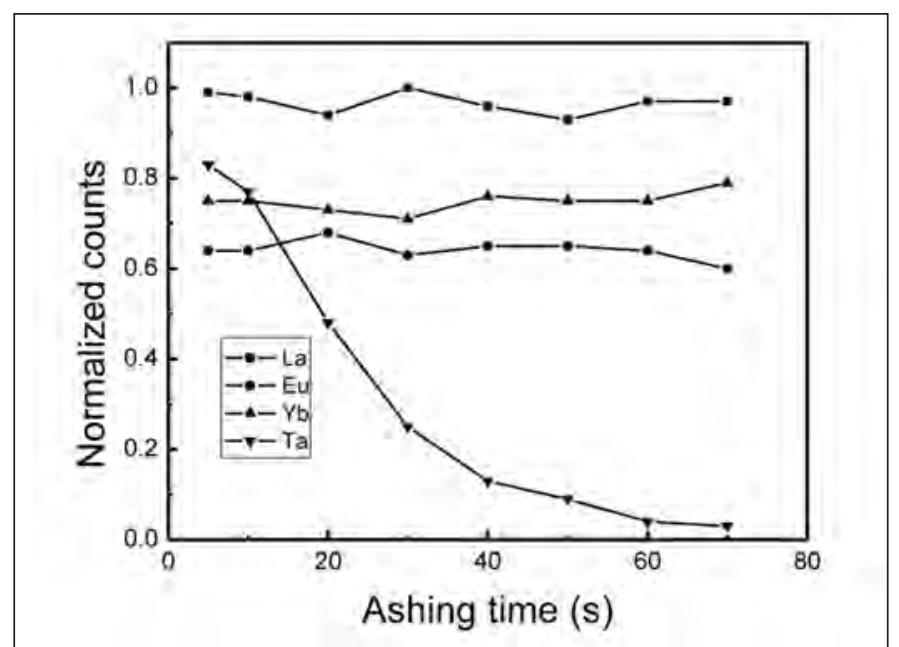

Fig. 3. Effect of ashing time on signal intensity. With PTFE: La, Eu, Yb and Ta, $3.0 \mathrm{ng} \mathrm{mL}^{-1}$.

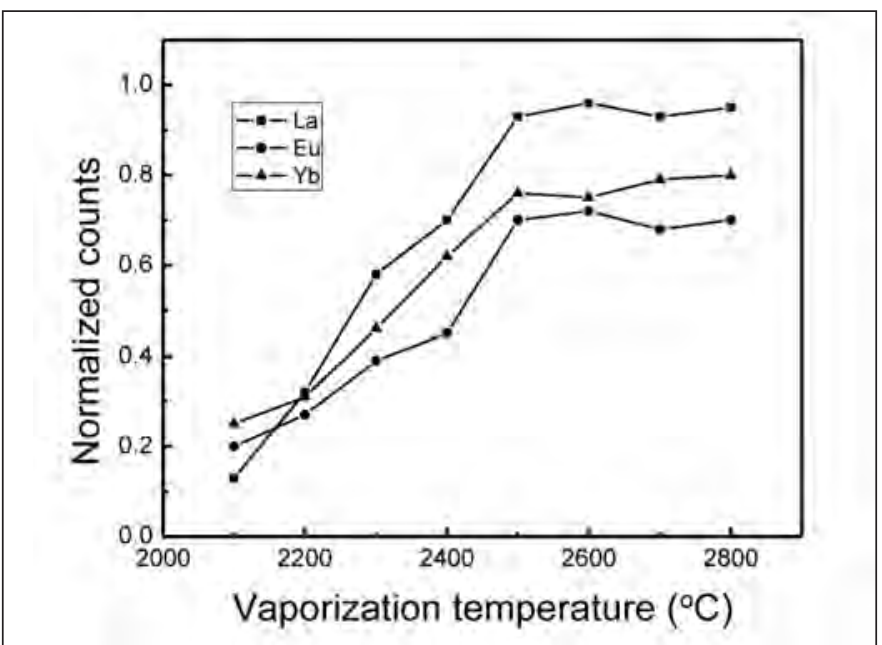

Fig. 4. Influence of vaporization temperature on signal intensity. With PTFE: La, Eu. and Yb, $3.0 \mathrm{ng} m L^{-1}$. plateau above a proper vaporization temperature (about $2500^{\circ} \mathrm{C}$ ). This can be due to the formation of the easily volatile fluorides characterized by similar vaporization behavior. In this study, a vaporization temperature of $2500{ }^{\circ} \mathrm{C}$ was employed for the simultaneous multielement determinations.

\section{Investigation of Matrix Effects}

The effects of the matrix concentrations on the determination of the REEs were studied by measuring the signal intensities for the solutions with variable concentrations of Ta (from 10 to $200 \mu \mathrm{g} \mathrm{mL}^{-1}$ ) and $3.0 \mathrm{ng} \mathrm{mL}^{-1}$ of the analytes. The experimental results show that, with PTFE as the chemical modifier, there is no apparent matrix effect for the analytes with a Ta concentration of $200 \mu \mathrm{g} \mathrm{mL} \mathrm{m}^{-1}$ at a pyrolysis temperature of $600^{\circ} \mathrm{C}$ and a pyrolysis time of 50 seconds. Thus, the selective volatilization is beneficial for decreasing the effect of the matrix on the analytes. With respect to the mechanism of matrix effect, further research work needs to be carried out.

\section{Influence of PTFE Amount}

The effect of PTFE concentration was examined by varying the PTFE percentage $(\mathrm{m} / \mathrm{v})$ from $0.20 \%$ to $2.0 \%$. The experimental results show that the signal intensities of the analytes increase with an increase in the PTFE concentration and reached a constant value at a PTFE concentration of about $1.0 \%$. Taking into account that the PTFE could be consumed by the matrix in a real sample analysis, a suitably higher PTFE concentration of $1.5 \%$ was used in subsequent studies.

\section{Detection Limits and Precision}

According to the definition of IUPAC, the detection limit (DL) is defined as the concentration corresponding to three times the standard deviation of the blanks. Under optimum conditions, the detection limits for $\mathrm{La}, \mathrm{Eu}$, and $\mathrm{Yb}$ were found to be $4.2,1.9$, and $3.5 \mathrm{ng} \mathrm{g}^{-1}$, respectively. The precision for $\mathrm{La}$, $\mathrm{Eu}$, and $\mathrm{Yb}$, expressed as relative standard deviations (RSDs), was $4.9,2.7$, and $4.1 \%$, respectively $\left(\mathrm{n}=9, \mathrm{c}=1.0 \mathrm{ng} \mathrm{mL} \mathrm{m}^{-1}\right)$. Calibration graphs of the analytes were linear over three orders of magnitude.

\section{Sample Analysis}

The proposed method was applied to the determination of La, $\mathrm{Eu}$, and $\mathrm{Yb}$ in $\mathrm{Ta}_{2} \mathrm{O}_{5}$ by the calibration curve method and the standard addition method. The same sample was also analyzed by PN (pneumatic nebulizer)-ICP-MS after removal of the matrix by a solvent extraction procedure (1). The analytical results obtained and summarized in Table II show good agreement.

\section{CONCLUSION}

The formation of the volatile fluorides not only improves the vaporization and transportation efficiency of the analytes, but also enhances the difference in the volatilities between the analytes and the matrix components. This difference can then be exploited for separating in situ the matrix from the analytes by selective vaporization.

The proposed method for the determination of trace REE impurities in $\mathrm{Ta}_{2} \mathrm{O}_{5}$ offers the following advantages: (a) less sample pretreatment, (b) small sample requirement, (c) simple and rapid 
TABLE II

Analytical Results of the Rare Earth Impurities in $\mathrm{Ta}_{2} \mathrm{O}_{5}(\mathrm{n}=5)$

\begin{tabular}{cccc}
\hline Element & $\begin{array}{c}\text { Calibration Curve } \\
\text { Method }\left(\mu \mathrm{g} \mathrm{g}^{-1}\right)\end{array}$ & $\begin{array}{c}\text { Standard Addition } \\
\text { Method }\left(\mu \mathrm{g} \mathrm{g}^{-1}\right)\end{array}$ & $\begin{array}{c}\text { PN-ICP-MS } \\
\left(\mu \mathrm{g} \mathrm{g}^{-1}\right)\end{array}$ \\
\hline $\mathrm{La}$ & $3.8 \pm 0.21$ & $4.0 \pm 0.37$ & $3.5 \pm 0.28$ \\
$\mathrm{Eu}$ & $1.1 \pm 0.09$ & $1.2 \pm 0.11$ & $1.0 \pm 0.06$ \\
$\mathrm{Yb}$ & $0.67 \pm 0.05$ & $0.71 \pm 0.12$ & $0.72 \pm 0.04$
\end{tabular}

a Analysis after matrix removal by solvent extraction.

operation, (d) reduction of sample contamination, and (e) no need for matrix matching. Therefore, this is likely to become an effective method for the determination of trace impurities in Ta-based powder samples.

\section{ACKNOWLEDGMENT}

This work was supported by the Nature Science Foundation and the Education Department Foundation of Hubei Province, in China.

$\overline{\text { Received November 13, } 2012 .}$

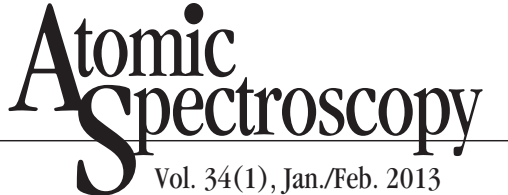

14. J. Kang, H. Chen, and X. Zeng, Spectrochim. Acta, Part B, 58(9), 1679 (2003).

15. E. Toda, A. Hioki, and M. Kubota, Anal. Chim. Acta 333(1-2), 51 (1996).

16. S. Chen and D. Lu, At. Spectrosc. 27(4), 128 (2006).

17. U. Schäffer and V. Krivan, Anal. Chem. 710, 849 (1999).

18. S. Chen, S. Zhu, and D. Lu, At. Spectrosc. 31(4), 117 (2010).

19. S. Tao, Y. Okamoto, and T. Kumamaru, Anal. Sci. 11, 319 (1995).

20 A.B. Volynsky and M.T.C. de LoosVollebregt, Spectrochim. Acta, Part B, 60(11), 1432 (2005).

21. F.G. Lepri, M.B. Dessuy, M.G. R.Vale, D.L.G. Borges, and B. Welz, Spectrochim. Acta, Part B, 61(8), 934 (2006).

22. S. Chen, H. Zhang, and B. Ruan, At. Spectrosc. 30(4), 143 (2009).

23. S. Chen, J. AOAC Intern. 90(1), 258 (2007). 